\title{
Autonomous Modular Optical Underwater Robot (AMOUR) Design, Prototype and Feasibility Study
}

\author{
Iuliu Vasilescu and Paulina Varshavskaya \\ Comp. Sci. and Artificial Intelligence Laboratory \\ Massachusetts Institute of Technology \\ Cambridge, Massachusetts, USA \\ \{iuliuv|paulina\}@csail.mit.edu
}

\author{
Keith Kotay and Daniela Rus \\ CSAIL \\ MIT \\ Cambridge, MA, USA \\ Dartmouth Robotics Laboratory \\ Dartmouth College \\ Hanover, NH, USA \\ \{kotay|rus\}@csail.mit.edu
}

\begin{abstract}
We propose a novel modular underwater robot which can self-reconfigure by stacking and unstacking its component modules. Applications for this robot include underwater monitoring, exploration, and surveillance. Our current prototype is a single module which contains several subsystems that later will be segregated into different modules. This robot functions as a testbed for the subsystems which are needed in the modular implementation. We describe the module design and discuss the propulsion, docking, and optical ranging subsystems in detail. Experimental results demonstrate depth control, linear motion, target module detection, and docking capabilities.
\end{abstract}

Index Terms-Modular robot, underwater robot, selfreconfiguring robot, optical ranging.

\section{INTRODUCTION}

We wish to develop small autonomous underwater robots that are modular and can establish ad-hoc underwater networks. Such robots will permit the exploration and monitoring of underwater environments, allowing applications such as long-term monitoring of underwater habitats, monitoring and surveillance of ports, modeling the impact of weather and ground activities (such as manufacturing and agriculture) on the water quality, and underwater geochemical prospecting. Each of these applications requires a long term underwater presence that can cover a large area and adapt to triggers in the environment, positioning and repositioning the robot or adjusting the sampling rate.

Our goal is to develop underwater modular autonomous robots and sensors that can function as teams of autonomous robots and mobile sensor networks. The modules we propose have uniform shape, can stack up, but have a range of different functionalities. Figures 1 and 2 show the concept of modular underwater robots that can deploy and recover sensor networks. Each robot has a computation, motor, buoyancy, and battery module, and an arbitrary number of sensor modules. ${ }^{1}$ A docking mechanism allows a module to attach to the one above it in the structure. Modules implement the discrete components of an underwater robot system such as buoyancy, propulsion, power, computation, and sensing. Each of these components will be present in a functioning robot but additional instances will increase the functionality of the robot. For example additional battery modules will extend its lifetime, and additional motor modules will increase the speed and maneuverability of the robot. This modular approach to underwater robots has several advantages. First, it provides versatility for the robot system, as the functionality of the robot is additive with respect to the modules in its body. Second, this is a fault tolerant approach to building underwater robots, as faulty modules can be eliminated from the system. Third, it supports the creation of underwater networks of robots and sensors that can use ad-hoc networking to provide adaptive sampling and coverage over larger spaces than those available to a single robot. Finally, the entire sensor network can be launched as one robot that could then do precision deployment and recovery of the individual nodes.

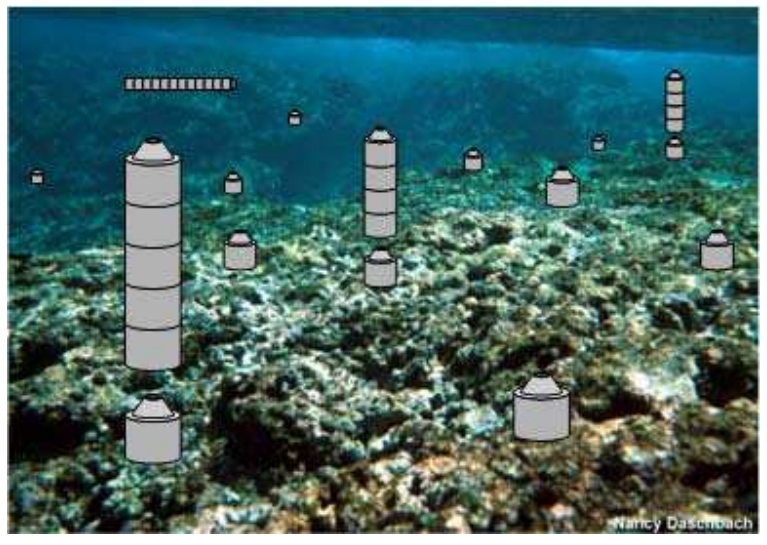

Fig. 1. Autonomous modular underwater vehicles deploying and collecting a sensor network. The proposed sensor modules stack up at the bottom of the underwater vehicle. They do not move on their own. One or more autonomous vehicles deploy the sensor modules by releasing them at desired locations. To collect a sensor, an autonomous vehicle docks with the sensor and connects it to its body.

\footnotetext{
${ }^{1}$ Separate sensor modules are not necessary if functional modules also contain the sensors to be deployed in the network.
} 


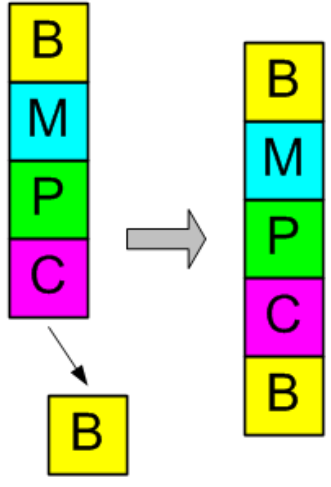

(a)

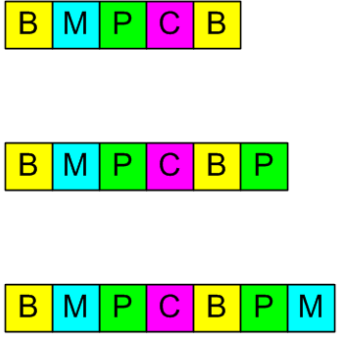

(b)

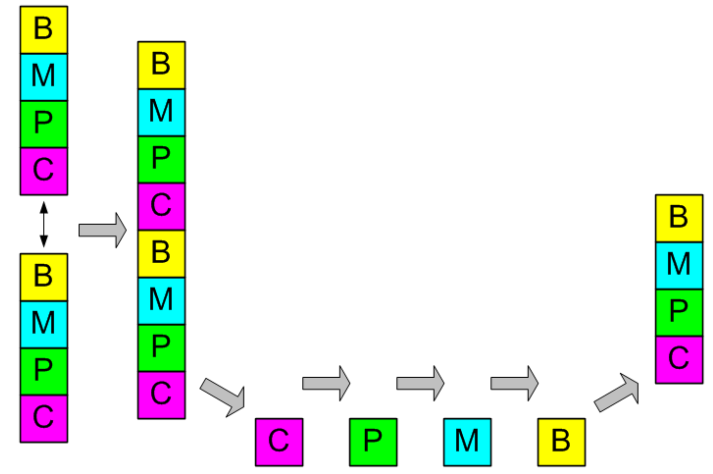

(c)

Fig. 2. Our concept is a modular system composed of a set of stackable, cylindrical modules with different functions. A working robot contains at least one of the four module types: buoyancy ('B'), motor ('M'), power ('P'), and computation ('C'). As shown in (a), a robot can dock with a module resting on the sea floor, adding that module to its configuration. Additional modules increase the capability of the robot. Adding a buoyancy module, (b) top, allows the robot to move horizontally with increased efficiency; adding an extra power module, (b) center, increases lifetime and thus autonomy; and an additional motor module, (b) bottom, increases maneuverability and speed. A module deployment sequence is shown in (c), with two robots docking, followed by the placement of the modules of the lower robot on the sea floor.

In this paper we describe a small underwater robot that was designed and built with the following goals:

- small, modular and scalable architecture

- ability to navigate and search for other robots

- ability to communicate with other modules, acting as a data mule in an underwater sensor network

- ability to dock with other modules, and

- ability to release other modules at designated places in the environment.

The robot we describe does not fully implement our modular underwater vision, but it demonstrates the key aspects of such a robot: autonomous control, navigation, communication, and docking. More specifically, we describe the architecture of the robot, we discuss its control and navigation abilities, we describe the optical communication system on the robot and its latching mechanism, and we show how these systems can be used for docking and release. Finally, we present experimental results testing all these component systems which are required for a fully modular architecture.

\section{RELATED WORK}

There has been much work in the fields of Autonomous Underwater Vehicles (AUVs), their control and navigation, underwater communications, sensors and docking [16]. In a 2000 survey [15], it was estimated that over 1,000 robotic underwater vehicles operate worldwide in industry, military and research applications. A small but growing portion of these are semi-autonomous or autonomous robots.

AUVs in general face severe navigation challenges due to the fact that water absorbs radio waves. There have been three types of navigation systems for autonomous robots underwater, which rely on different sensors: (1) dead-reckoning and inertial navigation, (2) acoustic, and (3) geophysical navigation [9]. Visual feature tracking has also been employed, in particular on the Kambara project
[13]. Others have found that combining sensor information, such as conventional long baseline acoustic sensing and Doppler effect, can improve underwater navigation [14].

Optical guidance systems, which give precise resolution at very short distances, have been used for the AUV's close-range homing and docking abilities. In [5] an optical quadrant tracker locks onto a light source for docking, as demonstrated underwater on a SeaGrant Odyssey IIB [3]. Alternatively, long baseline and ultra-short baseline acoustic beacons have also been used for docking [11].

Recently there has been interest in deploying multiple robots, in particular for oceanographic research applications. The Serafina project [8] explores large-scale formation control issues with multiple small, agile AUVs. Gliders such as Seaglider [6] are designed to dive to a programmed depth and resurface while taking measurements, moving for thousands of kilometers in a sawtooth pattern. Gliders have also been used in cooperative multi-AUV control research [7]. Such research is motivated by collaborative oceanographic research projects such as the Autonomous Ocean Sampling Network II [1]. It is becoming more important for the robots to be able to assist in the deployment or to act as parts of such large-scale data-collecting networks. A small submarine [4] has been proposed as a sensor in such a network. The robot houses a Mote sensor and can control its own depth.

In addition, some attempts have been made to create modular underwater robots. Inspired by eels [10] or lampreys [2], these are smaller-scale biomimetic robots whose modules are permanently joined in one configuration.

To the best of our knowledge, the present paper is a first attempt to describe a system which demonstrates through physical implementation the feasibility of all components required for a self-reconfiguring modular autonomous underwater robot. While the idea of such robots has recently been conceptualized, its physical implementation is still in 
a prototype stage. In particular in [12] controllers devised by genetic regulatory networks are applied to a simulated robot.

We have used insights developed in prior AUV research, in particular in our geophysical (compass-based) navigation system and our optical short-range homing system.

\section{ROBOT DESIGN}

Our goal is to develop a modular underwater robot which can self-reconfigure by stacking and unstacking its component modules. To accomplish this, four primary subsystems are required: (1) buoyancy control, (2) propulsion, (3) power, (4) and computation. We intend to segregate these subsystems into separate module types. A functional robot will contain at least one of each of these modules. Each module will have docking and communication capability, as well as a small onboard battery. The power module will contain several high capacity batteries and function as the primary power source for the robot. Modules will also have various sensors dependent on the module function. For example, the buoyancy control module will need a pressure sensor to monitor the robot depth and the computation module will have a compass for navigation control. All modules will use microprocessors for low-level control of their functions and for communication with other modules. The computation module, however, will have more powerful processing capability and will run a multitasking operating system such as Linux.

Our current implementation is a non-modular underwater robot which functions as a testbed for the various subsystems (see Fig. 3). The basic shape of the robot is a $38 \mathrm{~cm}$ tall acrylic cylinder with a $15 \mathrm{~cm}$ outside diameter and $14 \mathrm{~cm}$ inside diameter. The current prototype operates with a vertical orientation of the cylinder axis. This attitude is used for docking, since the modules stack end to end. We intend to operate the modular robot in both vertical and horizontal orientations, with the horizontal mode used for long distance movement of a complete robot due to the more streamlined shape. ${ }^{2}$

The cylinder is divided into three internal sections, with the top section containing the main microprocessor and batteries; the middle section containing the propulsion motors; and the lower section containing the optical ranging circuit board, the magnetic latch mechanism, and ballast. Currently, buoyancy control is dynamic although we intend to use a combination of dynamic and static buoyancy control on the modular system. The future buoyancy module will contain a ballast tank, water pump, and electrically actuated valves in addition to the buoyancy control microprocessor. Propulsion is provided by five thruster units: three dualshaft drives and two bow thruster units (see Fig. 3). All these off-the-shelf units are designed for use with $\mathrm{R} / \mathrm{C}$ model boats. The dual-shaft drives are mounted at an angle such that the DC motors are inside the robot and the shafts protrude through the side of the acrylic cylinder.

\footnotetext{
${ }^{2}$ Note that horizontal orientation requires two buoyancy modules, one near each end of the robot.
}



Fig. 3. Our current robot prototype which functions as a testbed for the various subsystems of our modular system including propulsion, optical ranging, and docking. The main housing is an acrylic cylinder with a $15 \mathrm{~cm}$ outside diameter. Two types of thrusters are employed: dual-shaft propeller drives ('A') and bow thrusters ('B'). The dual-shaft thruster propellers are shrouded to prevent them from coming into contact with external objects. This prototype does not have a latching probe on the upper cap.

These thrusters provide vertical movement and attitude control. The bow thrusters are mounted transversely and are used for lateral movement and turning. The motors are separated from all electronics inside the robot with two sealing partitions.

The docking mechanism is a probe and drogue system, with the top of each module containing the rod which functions as the probe and the bottom of each module featuring the conical drogue (see Fig. 4 and 5). Optical ranging is used to find potential modules with which to dock and as guides for the docking process. The lower compartment of our prototype contains the sensing photodiode array and the optical processing circuit board which must be near the photodiodes to reduce noise.

The subsystems will be discussed in detail in the following sections.

\section{A. Motor control and navigation}

The five thrusters on our robot provide full control over the six degrees-of-freedom of the robot. This is due to the angled orientation of the three dual-shaft thrusters, which allows them to affect multiple degrees of freedom. The result is a non-holonomic system, requiring multiple thrusters 


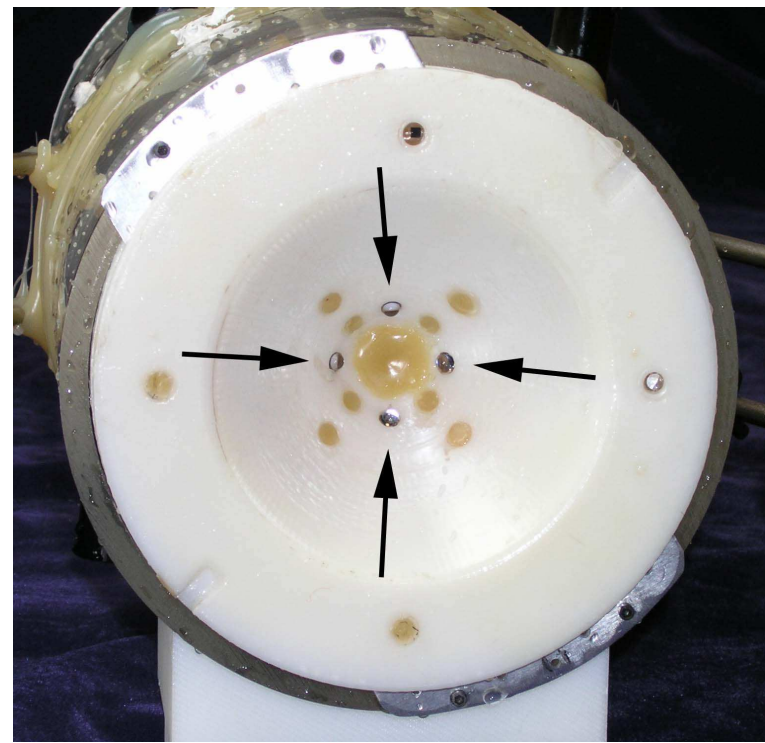

Fig. 4. The bottom cap of the robot has a conical indentation which functions as the drogue element of the probe and drogue docking system. The arrows indicate the four photodiodes which are used to obtain range and direction estimates for the target module.

to actuate simultaneously to generate single degree-offreedom control. In practice, attitude control is greatly affected by the position of the ballast in the lower section of the module. Thus, the robot has a strong tendency to remain upright in calm water. The primary function of the dualshaft thrusters, therefore, is vertical movement and dynamic buoyancy control. These movements require the opposed dual-shaft thrusters (' $\mathrm{A}_{1}$ ' and ' $\mathrm{A}_{3}$ ' in Fig. 3) to actuate simultaneously at the same speed. Rotation and lateral movement are controlled by the bow thrusters $\left(\mathrm{B}_{1}\right.$ and $\left.\mathrm{B}_{2}\right)$. Here again both thrusters are actuated simultaneously, with rotation requiring water flow in opposite directions through the thrusters and lateral movement requiring water flow in the same direction. Thus we can achieve the following control over the robot:

- long-distance travel While the robot is positioned horizontally, actuate opposing dual-shaft drives $\mathrm{A}_{1}$ and $\mathrm{A}_{3}$.

- local translation at given depth While the robot is upright, actuate bow thrusters $B_{1}$ and $B_{2}$ for water flow in the same direction.

- local rotation at given depth While the robot is upright, actuate bow thrusters $B_{1}$ and $B_{2}$ for water flow in the opposite direction.

- maintaining depth In an upright position, actuate opposing dual-shaft drives $\mathrm{A}_{1}$ and $\mathrm{A}_{3}$.

- vertical movement In an upright position, actuate opposing dual-shaft drives $\mathrm{A}_{1}$ and $\mathrm{A}_{3}$.

- tilt stabilization Actuate $A_{2}$ to balance the robot and control tilt.

Experimental results demonstrate excellent dynamic buoyancy control using a PD controller, and precise turning capability, as well as the ability to move in straight line using a compass for heading control (see Section IV).

The dual-shaft thrusters are fairly powerful units, capable of moving the robot at a maximum velocity of 1 meter per second along the axis of the cylinder. While this top speed is not used during our initial experiments, it is intended that the robot will move over long distances in a horizontal orientation. In contrast, the bow thrusters are relatively weak, and lateral movement is hampered by the larger cross sectional surface area. However, the bow thrusters are intended for small lateral motion corrections during docking, and therefore do not need to be as powerful as the main thrusters.

Out current prototype uses a digital compass to sense robot orientation. The compass heading information is used by a PID controller to adjust bow thruster power for straight-line motion. This works successfully in our test environment (an indoor swimming pool), but will not be sufficient in an ocean environment with currents and waves. We intend to add a GPS unit to our modular implementation, which will enable the robot to obtain precise location data at the surface. This data can be used to deploy modules in known locations. The deployed modules can then act as underwater beacons, allowing the robot to determine its position without surfacing.

The robot requires guidance beacons in order to find modules and dock to them. The process consists of four phases:

1) a long range module search using sound

2) short range robot guidance using light

3) module alignment using mechanical compliance, and

4) latching.

The long range search has not been implemented in our prototype, but will consist of an acoustic beacon system. The seeker robot will emit an acoustic signal which will be answered by the module, allowing the robot to home in on the module location. Since there are multiple module types, it is important for the robot to query for a specific module type, e.g., a battery module, a propulsion module, etc. Once the robot is within two meters of the target module the short range phase begins. ${ }^{3}$

\section{B. Optical ranging and communication}

The short range localization phase uses optical navigation, which has been implemented on our prototype robot. The process begins with the robot sending an optical message to a module resting on the sea floor, telling it to illuminate its optical beacon. Once the optical beacon is on, the robot uses its photodiode array to estimate the direction to the module (see Fig. 4). This information is used to guide the robot to a position directly above the module. The robot uses its bow thrusters controlled with a PID controller to accomplish this alignment phase.

Key aspects of the optical ranging system are the use of PIN photodiodes and high current LEDs. A PIN (positiveintrinsic-negative) photodiode has a large, neutrally doped

\footnotetext{
${ }^{3}$ When the robot is moving in close proximity to several modules on the sea floor, the long range phase may be bypassed. In this case, optical communication will be used to determine the module type and location.
} 
intrinsic region sandwiched between $\mathrm{p}$-doped and n-doped semiconducting regions. PIN photodiodes are much more sensitive than other photodiodes. High current LEDs are also very important, since the emitted light must be very intense to be visible in all ambient light conditions. A consequence of the DC restoration method described below is that the LED emission duty cycle is very low (2\%), allowing the LED power to be ten times greater than the continuous duty rating. This generates a very bright pulse of light for a short period of time.

1) DC restoration method: A key problem in implementing underwater optical ranging is interference from ambient light. It is necessary for the robot to distinguish the module's optical beacon in the presence of various lighting conditions, including direct sunlight. In addition, the character of the light may be constantly changing due to waves, clouds, fish, etc. On land it is possible to use a frequency of light that is not present in the environment, however this is not possible in water since most frequencies are severely attenuated. The remaining frequencies are those that are present in sunlight and therefore can interfere with optical ranging. Furthermore, it is desirable that the range of signal detection be maximized, restricting the usable frequency range to those that have minimum attenuation.

Our solution to this problem is the use of a synchronized pulse train with pre-pulse determination of the ambient light level for comparison with the light level at the expected pulse time. This method is similar to that of the DC restoration circuit used in television receivers to adjust the black level regardless of the brightness of the rest of the image. In our system, a $20 \mu$ s optical pulse is generated every $1 \mathrm{~ms}$. The receiver synchronizes itself to this pulse train and "samples" the ambient light just prior to an expected pulse. The sampling is done by charging a capacitor to the output voltage of a photodiode. This voltage is then compared to the actual voltage at the time of the expected pulse to obtain the effective difference between the ambient light and the ambient light + beacon signal. The difference is then amplified and converted to a digital value. This process is performed simultaneously using multiple photodiodes oriented at 30 degrees from the vertical plane which allows the robot to estimate the angle to the optical beacon (see Fig. 4 for the photodiode array on our current prototype).

\section{Docking}

To join to robot modules together, one robot aligns itself vertically above the other module, henceforth known as the base module. When all four photodiodes receive a signal of equal strength, the two opposing dual-shaft thrusters $A_{1}$ and $\mathrm{A}_{3}$ are actuated, and the robot moves down to land on the base module. The alignment during landing is aided by the probe and drogue configuration of the connecting sides, as seen in Fig. 5. The bottom cap of the robot is shaped as a cone, which guides the base probe into place as the robot lands. At the apex of the cone a latch plate with a variable width hole moves freely in the horizontal plane in a tublike compartment. The plate is attached on one side to a

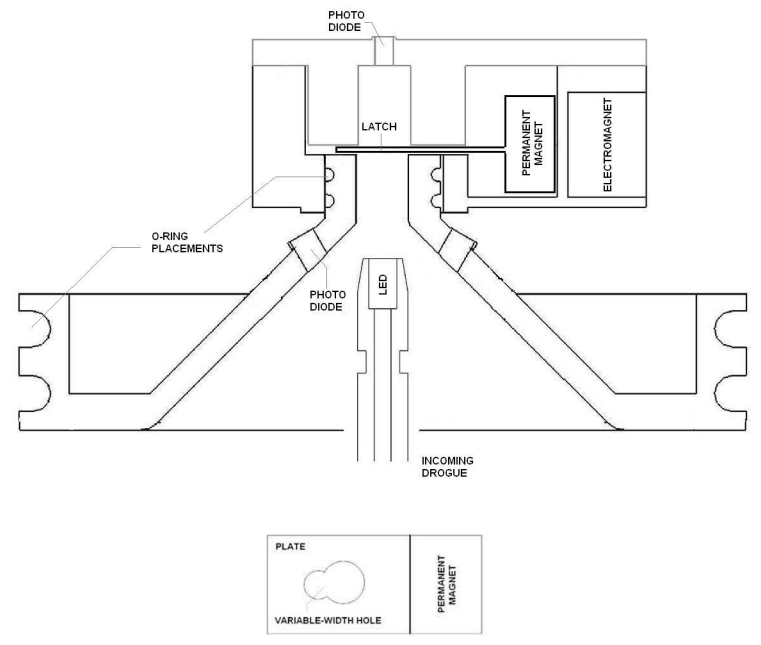

Fig. 5. The mechanics of the docking and latching mechanism in crosssection. The latching plate with a variable-width hole, viewed from above.

permanent magnet. An electromagnet is mounted coaxially with the permanent magnet behind a thin waterproof wall.

When the robot is ready to dock, a current is sent to the electromagnet which then repels the magnetic latch. The larger diameter hole is thus positioned above the apex of the cone. The probe can then enter and is latched in place by temporarily reversing the polarity of the electromagnet to boost the attraction between the magnets. The attractive force at rest is enough to keep the probe latched.

The magnetic latching mechanism is first triggered when the photodiode array suddenly stops sensing light after returning maximum values for a short period of time. This indicates that the probe has entered the drogue. At that time, the single photodiode mounted above the variablewidth hole in the latching plate will sense the light emitted from the probe LED.

\section{EXPERIMENTS}

We have conducted a number of experiments in water to demonstrate feasibility of the required components. The experimental environments range from a $0.5 \mathrm{~m}^{2}, 1$ meter deep test tank in our laboratory space to a 23 meter swimming pool with a deep end of 4 meters. All experiments were performed in autonomous mode.

1) Compass navigation: The robot used its digital compass to navigate in a straight line across a small swimming pool. We have found that the robot reliably maintained direction during eight trials and completed the 5 meter traverse in approximately 40 seconds each time. Figure 6 shows the robot during such a small pool trial.

2) Depth control, optical navigation and landing: In a series of experiments in a small swimming pool, the robot performed the tasks of maintaining a range of specified depths over trials of 15 minutes each, finding a lightemitting base and docking to it. ${ }^{4}$ The robot demonstrated

\footnotetext{
${ }^{4}$ In these trials, the latching mechanism was not activated. The robot only landed on the base.
} 


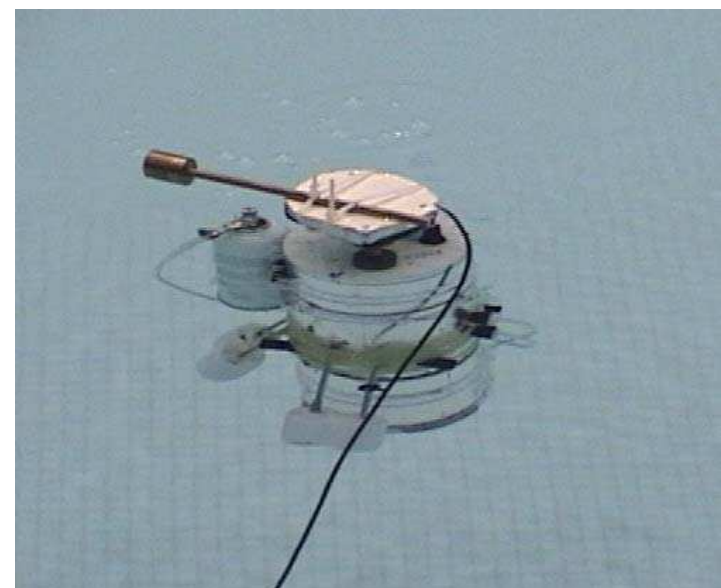

Fig. 6. The robot in a small swimming pool during an experiment in compass-based navigation.

an ability to perform all of these tasks. It was then taken to a 23 meter swimming pool at the deep end, where it succeeded at diving in a controlled fashion until it could perceive the light from the base module, locating the module and docking to it. Experiments show that the robot can maintain depth in the range from surface to 4 meters with a resolution of approximately $5 \mathrm{~cm}$.

3) Optical communication: The robot communicated with a module placed at the bottom of the test tank. Oneway communication was tested, which allows the robot to act as a data mule. The robot can communicate with a module from a distance of 2 meters at a maximum angle of 30 degrees. We used 20-byte messages with checksums and observed a baud rate of approximately 1 kbit per second.

4) Docking with latching: The robot docked to a light-emitting base module, latched onto its probe and picked up the module during a series of experiments performed in the test tank at a depth of 1 meter.

Both the robot and the experiments we run are a work in progress. For the immediate future, we are focusing on collecting quantitative data on each aspect of the robot's abilities and on demonstrating reliable success.

\section{DISCUSSION AND FUTURE WORK}

We have reported on the design and implementation of a first prototype robot, intended as a testbed for our ongoing project in creating a self-reconfiguring, modular underwater robot. The hardware implementation consists of a single cylindrical module which incorporates the motor, battery, and CPU subsystems as well as the latching mechanism. This system has allowed us to test the essential components of the future modular system. We have presented experimental results which suggest that our robot is capable of compass-based navigation, optical homing and communications, docking with a module resting on the floor of a swimming pool, and traveling while latched to a module.
Our goal for the immediate future is to develop a modular underwater robot which is capable of self-reconfiguring for the purpose of deploying and collecting the component modules. Proposed module types are motor, buoyancy, battery, and CPU. Just as on our current prototype, modules will be cylindrical and stack along the axis of of the cylinder, using the same latching probe and drogue docking mechanism. The modules will be equipped with sensors, allowing a deployed module array to function as a sensor network.

We will then focus on developing control algorithms for this robot, which will allow modules to cooperate in solving underwater tasks such as motion control given changing inertial characteristics, navigation, deployment and recovery. We plan to conduct extensive experiments in a test tank and in shallow ocean waters.

We also described advantages of our modular design, such as versatility, precision deployment, fault tolerance, and networking capabilities. However, modularity in general, and the current stacking design in particular, present certain issues which will need to be addressed in future work.

1) Changing inertial characteristics: As the robot picks up more modules, their weight will contribute to changes in the robot's mass distribution and therefore its inertial characteristics in water. Control algorithms will need to adapt to these changes. Since modules can communicate and sense the presence of neighbors, the CPU can determine the robot's exact composition at any time and modify the control law accordingly.

2) Reliability of serial stacking and release: Our current design calls for a serial stacking of modules - a configuration that is potentially vulnerable to specific faults. For example, if all power is transmitted from the bottom of the stack (battery module), then any faulty module in between can prevent its upward neighbors from receiving power. A modular architecture, even a serially stackable one, can actually be more fault-tolerant in such a situation. An additional battery module placed upward of the faulty one can provide power to otherwise cut-off neighbors. We envisage an operation with a considerable number of modules, such that robots may carry more instances of every type to increase their functionality and faulttolerance.

Another potential problem is docking mechanism failure. If the latch fails, it may be possible to release a mini-stack consisting of a working module on top of the faulty one. It may also be possible to send another robot to the same location for another attempt at release, or to pull at the other end of the faulty module in order to disconnect the latch. In this case again, working with a large number of modules increases our chances of successful operation.

3) Using known module positions for navigation: Errors in precision deployment and error accumulation over time (e.g, drift) can present problems for beacon navigation using pre-positioned modules. The severity of this problem will depend on the mission of the robot as 
well as on the redundancy in the network of beacons. It will need to be addressed on the application-dependent, algorithmic level.

To implement a working undersea sensor network, our modules will need to operate robustly in rough sea waters and on the sea bed, which has considerable relief, sand, and vegetation. Such an environment may obscure or overwhelm the sensors, and make navigation and docking more challenging. However, these observations will be of more concern during the final stages of the project, when we develop a large scale network capable of ocean operation, as part of a specific scientific application. Before such an undertaking becomes possible, we need to address issues more fundamental to robotic control and computer science.

\section{ACKNOWLEDGMENT}

Support for this work was provided through NSF awards IRI-9714332, EIA-9901589, IIS-9818299, IIS-9912193 and EIA-0202789, ONR award N00014-01-1-0675, Intel, and MIT's project Oxygen. We are grateful for this support.

\section{REFERENCES}

[1] Autonomous Ocean Sampling Network (AOSN)II, collaborative project. http://www.princeton.edu/d̃csl/aosn/.

[2] J. Ayers, C. Wilbur, and C. Olcott. Lamprey robots. In Proceedings of the International Symposium on Aqua Biomechanisms, 2000.

[3] J. G. Bellingham, C. A. Goudey, T. R. Consi, J. W. Bales, D. K. Atwood, J. J. Leonard, and C. Chryssostomidis. A second generation survey AUV. In IEEE Conference on Autonomous Underwater Vehicles, Cambridge, MA, USA, 1994.

[4] Vitaly Bokser, Carl Oberg, Gaurav S. Sukhatme, and Aristides A. Requicha. A small submarine robot for experiments in underwater sensor networks. In International Federation of Automatic Control Symposium on Intelligent Autonomous Vehicles, 2004.

[5] Steve Cowen, Susan Briest, and James Dombrowski. Underwater docking of autonomous undersea vehicles using optical terminal guidance. In Oceans97, MTS/IEE Conference, 1997.
[6] Charles C. Eriksen, T. James Osse, Russel D. Light, Timothy Wen, Thomas W. Lehman, and Peter L. Sabin. Seaglider: A long-range autonomous underwater vehicle for oceanographic research. IEEE Journal of Oceanic Engineering, 26(4):424-436, October 2001.

[7] E. Fiorelli, N. E. Leonard, P. Bhatta, D. Paley, R. Bachmayer, and D. M. Fratantoni. Multi-AUV control and adaptive sampling in Monterey Bay. In Proceedings of the IEEE Autonomous Underwater Vehicles: Workshop on Multiple AUV Operations, Sebasco, ME, USA, June 2004.

[8] S. Kalantar and U. R. Zimmer. Contour shaped formation control for autonomous underwater vehicles using canonical shape descriptors and deformable models. In Proceedings of the IEEE International Conference on Marine Technology and Ocean Science, Kobe, Japan, 2004.

[9] John J. Leonard, Andrew A. Bennett, Christopher M. Smith, and Hans J. S. Feder. Autonomous underwater vehicle navigation. Memorandum 98-1, MIT Marine Robotics Laboratory, Cambridge, MA, USA, 1998.

[10] Ken McIsaac and Jim Ostrowski. A geometric approach to anguilliform locomotion: Modelling of an underwater eel robot. In Proceedings of the IEEE Conference of Robotics and Automation, pages 2843-2848, 1999.

[11] H. Singh, M. Bowen, F. Hover, P. LeBas, and D. Yoerger. Intelligent Docking for an Autonomous Ocean Sampling Network. In Oceans97, MTS/IEE Conference, Halifax, October 1997.

[12] Tim Taylor. A genetic regulatory network-inspired real-time controller for a group of underwater robots. In Proceedings of the 8th Conference on Intelligent Autonomous Systems, pages 403-412, Amsterdam, 2004.

[13] D. Wettergreen, C. Gaskett, and A. Zelinsky. Autonomous control and guidance for an underwater robotic vehicle. In Proceedings of the International Conference on Field and Service Robotics, Pittsburgh, USA, September 1999.

[14] L. L. Whitcomb, D. R. Yoerger, and H. Singh. Combined Doppler/LBL based navigation of underwater vehicles. In Proceedings of the 11th International Symposium on Unmanned Untethered Submersible Technology, Durham, NH, USA, August 1999.

[15] Louis L. Whitcomb. Underwater robotics: Out of the research laboratory and into the field. In Proceedings of the IEEE International Conference on Robotics and Automation, pages 709-716, April 2000.

[16] Junku Yuh. Design and control of autonomous underwater robots: A survey. Autonomous Robots, 8(1):7-24, 2000. 